\title{
Insights into Social Perception in Autism
}

\author{
PPunit Shah and Sophie Sowden \\ MRC Social, Genetic and Developmental Psychiatry Centre, Institute of Psychiatry, Psychology, \& Neuroscience, King's College London, University of \\ London, London, SE5 8AF, United Kingdom \\ Review of Cusack et al.
}

The perception of human movement, such as facial expressions and other bodily actions, is critically important for interpreting social cues. It is therefore not surprising that research into action perception-typically through the measurement of biological motion processing-has garnered considerable research attention (Blake and Shiffrar, 2007). Biological motion processing is governed by several brain regions working in concert with posterior regions of superior temporal sulci (pSTS), and it is considered a hallmark of typical social cognition (Pavlova, 2012). Conversely, impaired biological motion perception has been posited as a characteristic feature of Autism Spectrum Disorder (ASD), consistent with the social difficulties observed in this neurodevelopmental disorder (Kaiser and Pelphrey, 2012). However, theoretical interest in abnormal biological motion perception has perhaps outpaced empirical evidence, and existing research on action perception in ASD is highly inconsistent. In a detailed investigation published in The Journal of Neuroscience, Cusack et al. (2015) examined confounding factors in the literature

Received March 25, 2015; revised April 26, 2015; accepted April 29, 2015. P.S. and S.S. are supported by doctoral studentships from the Medical Research Council. We are very grateful to Geoffrey Bird and Francesca Happé for their comments on an earlier version of the manuscript.

The authors declare no competing financial interests.

Correspondence should be addressed to either Punit Shah or Sophie Sowden, MRC Social, Genetic and Developmental Psychiatry Centre, Institute of Psychiatry, Psychology, \& Neuroscience, King's College London, University of London, London, SE5 8AF, UK. E-mail: punit.shah@kcl.ac.uk or sophie.sowden@kcl.ac.uk.

DOI:10.1523/JNEUROSCI.1216-15.2015

Copyright $\odot 2015$ the authors $\quad 0270-6474 / 15 / 358689-02 \$ 15.00 / 0$ and devised methodological innovations to investigate action perception in ASD.

Action processing is commonly studied using moving points of light to represent human bodies in motion, and atypical processing of upright point-light displays has been taken as evidence for impaired action perception in ASD. However, these results might be attributable to deficient executive function and/or holistic processing in ASD (Behrmann et al., 2006). Cusack et al. (2015) identified the use of an inversion condition, in which point-light displays are presented upsidedown, as critical to control for these potential confounds. Upright moving faces and bodies are perceived holistically as non-decomposable wholes. This perception is disrupted in the presence of increasing amounts of visual noise and when stimuli are inverted. The accuracy with which inverted images are perceived is further disrupted by visual noise. Importantly, the ratio between an individual's sensitivity to upright versus inverted point-light displays provides a measure of biological motion perception that is not confounded by differences in executive functioning and holistic processing (Neri et al., 2007).

Inconsistent research on action perception in ASD is likely to stem from different aspects of action perception being studied independently of one another, and often using different stimuli. In contrast to previous research, Cusack and colleagues (2015) recognized that action perception involves many components, such as detecting and then distinguishing between actions observed in biological motion from those observed in nonbiological motion, and differentiating robotic from natural limb movements. It also involves discriminating between more complex action (e.g., fighting and dancing), the integration of moving limbs into a whole body, and interpreting whether two bodies are moving in synchrony or not. Cusack et al. (2015) therefore performed six psychophysical experiments using point-light displays, measuring biological motion perception for several components of action perception, comparing autistic participants with a matched group of typically developing controls.

In addition to the aforementioned features of the study design, attention was given to ensure the testing environment was suitable for people with (and without) ASD completing lengthy experiments (e.g., suitable rooms with which they were familiar). Although this is often overlooked, or at least not reported by researchers, Cusack and colleagues (2015) have raised standards in this domain. More generally, the study was well conducted and the results of the experiments were clear.

In line with previous work, typically developing participants exhibited robust action perception and, where appropriate, showed inversion effects - that is, biological motion processing was reduced on upside-down trials. Critically, the ASD group showed the same pattern of results across six experiments, demonstrating sensitivity to biological motion perception for several different types of action, 
indicative of intact action perception. These findings follow an increasing number of studies showing-by carefully designing stimuli, implementing appropriate control conditions, and minimizing extraneous cognitive demands - that several aspects of social perception are intact in ASD (e.g., social orienting, facial adaptation; Shah et al., 2013; R. Cook et al., 2014a). Although it is clearly important to establish which social abilities are aberrant in people with ASD, understanding which functions are intact can equally contribute to the design of cognitive-behavioral interventions to improve functioning and quality of life.

These null results and small sample sizes ( $n=15$ to 18 ) may lead to questions regarding statistical power because the group difference in sensitivity to upright point-light displays approached significance. However, this (suboptimal) measure of biological motion processing is confounded with differences in holistic processing and executive functioning. Cusack et al.'s (2015) experimental design mitigates this concern because the overall group difference in the optimal measure of biological motion processing (i.e., upright/inversion ratio) was not close to significance, and the effect size of the group difference was negligible. This pattern of results highlights a broader issue that small samples in clinical cognitive neuroscience are not problematic in and of themselves. Rather, theoretically grounded experimental designs can reduce the need for large samples to meaningfully interpret data (see also Bacchetti et al., 2011). In contrast, however, psychophysical data analysis is made difficult from noisy data generated by ASD participants, which required the authors to analyze a small subset of responses to interpret their results. Given the interindividual variability expected in heterogeneous ASD samples, it may have been prudent to recruit a larger sample. On a related note regarding sample characteristics, it is to be emphasized that the study was conducted in adolescents and, as Cusack et al. (2015) acknowledge, it is likely that action perception follows a developmental trajectory. Therefore, studies of equal rigor in both children and adults with ASD, or better yet, a longitudinal follow-up of the same participants, will be of exceptional interest. The foregoing issues do not call into question the conclusions of the study, but follow-up work will benefit from a richer sample, both in terms of size and age range.
Cusack and colleagues (2015) made a concluding remark that, although their action perception is intact, individuals with ASD may not respond appropriately in typical social interaction. They did not measure participants' motoric responses, but given that action perception precedes action imitation, their findings can be further situated in the literatures on imitation and movement kinematics in ASD. This is because the neural loci (e.g., pSTS) involved in action perception are believed to have bidirectional projections to the mirror neuron system, a network of brain regions seemingly involved in action recognition and imitation (for a recent discussion, see R. Cook et al., 2014b). Indeed, impaired imitation was once regarded as a defining feature of ASD (Williams et al., 2001), which, where reported, has often been attributed to aberrant action perception. However, Cusack and colleagues' (2015) data speak against this possibility. Instead, their results accord with evidence that automatic imitation is intact in ASD (Bird et al., 2007) and that top-down modulation of social perception (Cook et al., 2012) and control of social behaviors (Sowden and Shah, 2014) are atypical in this population. Similarly, Cusack et al. (2015) made only a cursory reference to recent reports that people with ASD show atypical kinematic profiles-for example, moving with greater acceleration and velocity than typical controls (J. Cook et al., 2013, 2014). More pertinent in relation to Cusack et al.'s (2015) research are findings from these studies that atypical kinematics can affect action perception and that this is associated with symptom severity in ASD. This intriguing work is still in its infancy, but now that Cusack and colleagues (2015) have established that 'passive' perception of action is intact in ASD, future work should focus on 'active' action perception while individuals are engaged in movement and social interactions.

In summary, Cusack and colleagues (2015) designed a series of well controlled experiments to investigate action perception in ASD. Consideration of sample characteristics notwithstanding, the study makes a clear contribution to an otherwise mixed literature on social perception in ASD: Cusack et al.'s (2015) results demonstrated that autistic individuals show intact action perception. This follows growing evidence that many aspects of social perception and action imitation are intact in individuals with ASD. Follow-up studies in this field of research will profit from using Cusack et al.'s (2015) method- ology within a developmental framework and elucidating the role of movement kinematics on action perception during social interaction.

\section{References}

Bacchetti P, Deeks SG, McCune JM (2011) Breaking free of sample size dogma to perform innovative translational research. Sci Transl Med 3:87ps24. CrossRef Medline

Behrmann M, Thomas C, Humphreys K (2006) Seeing it differently: visual processing in autism. Trends Cogn Sci 10:258-264. CrossRef Medline

Bird G, Leighton J, Press C, Heyes C (2007) Intact automatic imitation of human and robot actions in autism spectrum disorders. Proc $\mathrm{R}$ Soc B 274:3027-3031. CrossRef Medline

Blake R, Shiffrar M (2007) Perception of human motion. Annu Rev Psychol 58:47-73. CrossRef Medline

Cook J, Barbalat G, Blakemore SJ (2012) Topdown modulation of the perception of other people in schizophrenia and autism. Front Hum Neurosci 6:175. CrossRef Medline

Cook J, Blakemore SJ, Press C (2013) Atypical basic movement kinematics in autism spectrum conditions. Brain 136:2816-2824. CrossRef Medline

Cook J, Swapp D, Pan X, Bianchi-Berthouze N, Blakemore SJ (2014) Atypical interference effect of action observation in autism spectrum conditions. Psychol Med 44:731-740. CrossRef Medline

Cook R, Brewer R, Shah P, Bird G (2014a) Intact facial adaptation in autistic adults. Autism Res 7:481-490. CrossRef Medline

Cook R, Bird G, Catmur C, Press C, Heyes C (2014b) Mirror neurons: from origin to function. Behav Brain Sci 37:177-192. CrossRef Medline

Cusack JP, Williams JH, Neri P (2015) Action perception is intact in autism spectrum disorder. J Neurosci 35:1849-1857. CrossRef Medline

Kaiser MD, Pelphrey KA (2012) Disrupted action perception in autism: behavioral evidence, neuroendophenotypes, and diagnostic utility. Dev Cogn Neurosci 2:25-35. CrossRef Medline

Neri P, Luu JY, Levi DM (2007) Sensitivity to biological motion drops by approximately $1 / 2$ log-unit with inversion, and is unaffected by amblyopia. Vision Res 47:1209-1214. CrossRef Medline

Pavlova MA (2012) Biological motion processing as a hallmark of social cognition. Cereb Cortex 22:981-995. CrossRef Medline

Shah P, Gaule A, Bird G, Cook R (2013) Robust orienting to protofacial stimuli in autism. Curr Biol 23:R1087-R1088. CrossRef Medline

Sowden S, Shah P (2014) Self-other control: a candidate mechanism for social cognitive function. Front Hum Neurosci 8:789. CrossRef Medline

Williams JH, Whiten A, Suddendorf T, Perrett DI (2001) Imitation, mirror neurons and autism. Neurosci Biobehav Rev 25:287-295. CrossRef Medline 